\title{
Theory of dendritic growth in the presence of lattice strain.
}

\author{
D. Pilipenko, E. A. Brener, and C. Hüter \\ Institut für Festkörperforschung, Forschungszentrum Jülich, D-52425 Jülich, Germany
}

(Dated: November 13, 2018)

\begin{abstract}
Elastic effects due to lattice strain modify the local equilibrium condition at the solid-solid interface compared to the classical dendritic growth. Both, the thermal and the elastic fields are eliminated by the Green's function techniques and a closed nonlinear integro-differential equation for the evolution of the interface is derived. In the case of pure dilatation, the elastic effects lead only to a trivial shift of the transition temperature while in the case of shear transitions, dendritic patterns are found even for isotropic surface energy.

PACS numbers: $68.70 .+\mathrm{w}, 81.10 . \mathrm{Aj}, 89.75 . \mathrm{Kd}$
\end{abstract}

Solvability theory has been very successful in predicting certain properties of dendritic growth and a number of related phenomena (see, for example, $[1,2]$ ). The solution of the two-dimensional steady state growth is described by a needle crystal, which is assumed to be close in shape to the parabolic Ivantsov solution [3]. If anisotropic capillary effects are included, a single dynamically stable solution is found for any external growth condition. This theory has also been extended to the three-dimensional case $[4,5]$. The capillarity is a singular perturbation and the anisotropy of the surface energy is a prerequisite for the existence of the solution. In the case of isotropic surface energy, dendritic solution does not exist and instead the so-called doublon structure is the solution of the problem [6, 7].

Usually, the structural phase transitions in solids are accompanied by small lattice distortions leading to elastic deformations (for a review, see [8, 9]). One of the well-known consequences is a thermodynamic elastic hysteresis-i.e., the splitting of the phase equilibrium point into two points: the point of the direct and the inverse transition. This is mainly due to the coherency at the interface boundary, meaning that the lattice layers remain continuous through the boundary. Correspondingly, the hysteresis disappears without interface coherence [10, 11]. However, the systematic investigation of the growth $k i$ netics of such phase transitions is much less developed. Recently, the pattern formation processes controlled by interface kinetics have been considered in [12] and the growth of spherical inclusions under elastic and thermal influence were investigated in [13] by means of the phasefield model.

In this Letter, we discuss the influence of elastic strain on dendritic growth in solids controlled by heat diffusion. Significant progress in the description of dendritic growth was made by the elimination of the thermal field using the Green's function technique. This allows to obtain a closed equation for the interface evolution (see for example [14]). The crucial point of the present analysis is that the elastic field can also be eliminated by the corresponding Green's function technique. By these means, we derive here, as in the classical dendritic growth the- ory, a single integro-differential equation for the shape of the interface which takes into account elastic effects. Then, we consider two simple examples of dilatational and shear transformations. We show that in the case of a pure dilatation, the elastic effects lead only to a trivial shift of the transition temperature. However, for the case of shear transitions, we find dendritic patterns even without the anisotropy of the surface energy required by the classical dendritic growth theory. In this sense, the elastic effects serve as a new selection mechanism.

Thermodynamics of the model. Let us consider the growth of a new $\beta$-phase inside of an unbounded mother $\alpha$-phase. We denote the characteristic lattice strain (also known as the stress-free strain tensor), associated with the phase transition, by $\epsilon_{i k}^{0}$. The free energy density in the initial $\alpha$-phase is

$$
F_{\alpha}=F_{\alpha}^{0}(T)+\frac{\lambda}{2} \epsilon_{i i}^{2}+\mu \epsilon_{i k}^{2}
$$

where $F_{\alpha}^{0}(T)$ is the free energy density without elastic effects, which depends only on the temperature $T, \epsilon_{i k}$ are the components of the strain tensor, and $\lambda$ and $\mu$ are the elastic moduli of isotropic linear elasticity. The free energy density of the growing $\beta$-phase is given by:

$$
F_{\beta}=F_{\beta}^{0}(T)+\frac{\lambda}{2}\left(\epsilon_{i i}-\epsilon_{i i}^{0}\right)^{2}+\mu\left(\epsilon_{i k}-\epsilon_{i k}^{0}\right)^{2} .
$$

Here, we neglect the difference between the elastic coefficients in the two phases. We also assume that the elastic effects are small i.e., $\epsilon_{i k}^{0} \ll 1$. Since in our description the reference state for both phases is the undeformed initial phase, the coherency condition at the interface reads $u_{i}^{(\alpha)}=u_{i}^{(\beta)}$, where $u_{i}$ is the displacement vector. Mechanical equilibrium at the interface demands $\sigma_{n n}^{(\alpha)}=\sigma_{n n}^{(\beta)}$ and $\sigma_{n \tau}^{(\alpha)}=\sigma_{n \tau}^{(\beta)}, \sigma_{n s}^{(\alpha)}=\sigma_{n s}^{(\beta)}$. Here indices $n$ and $(\tau, s)$ denote the normal and tangential directions with respect to the interface; the stress tensor is defined as:

$$
\sigma_{i k}=\frac{1}{2}\left(\frac{\partial F}{\partial \epsilon_{i k}}+\frac{\partial F}{\partial \epsilon_{k i}}\right)
$$


The condition of phase equilibrium requires the continuity of a new potential

$$
\tilde{F}=F-\sigma_{n n} \epsilon_{n n}-2 \sigma_{n \tau} \epsilon_{n \tau}-2 \sigma_{n s} \epsilon_{n s}
$$

across the flat interface [15], which takes into account the coherency constraint. In the general case of curved interfaces, the surface energy $\gamma$ has also to be incorporated, and the phase equilibrium condition for each interface point in the case of isotropic surface energy reads

$$
\tilde{F}_{\alpha}-\tilde{F}_{\beta}-\gamma \kappa=0
$$

where $\kappa$ is the local curvature of the interface.

Solution of the elastic problem. Let us denote by $\tilde{\sigma}_{i k}$ the stress tensor which is related to the strain field $\epsilon_{i k}$ by the usual Hooke's law. Then, the stress in the new $\beta$ phase can be written as $\sigma_{i k}^{(\beta)}=\tilde{\sigma}_{i k}^{(\beta)}-\sigma_{i k}^{0}$, while it remains unchanged in the mother $\alpha$-phase: $\sigma_{i k}^{(\alpha)}=\tilde{\sigma}_{i k}^{(\alpha)}$. The tensor $\sigma_{i k}^{0}$ is related to the lattice strain $\epsilon_{i k}^{0}$ by Hooke's law

$$
\sigma_{i k}^{0}=\frac{E}{1+\nu}\left(\epsilon_{i k}^{0}+\frac{\nu}{1-2 \nu} \delta_{i k} \epsilon_{l l}^{0}\right),
$$

where $E$ is the Young's modulus and $\nu$ is the Poisson ratio. Thus, the mechanical equilibrium conditions at the interface require us to introduce the interface force density in the equilibrium equation $\partial \tilde{\sigma}_{i k} / \partial x_{k}=f_{i}$,

$$
f_{i}=\left(\tilde{\sigma}_{i k}^{(\beta)}-\tilde{\sigma}_{i k}^{(\alpha)}\right) n_{k}=\sigma_{i k}^{0} n_{k},
$$

where the normal vector $\mathbf{n}$ points from the $\beta$-phase into the $\alpha$-phase. Because the forces act only at the interface, the displacement field can be written as an integral over the interface surface:

$$
u_{i}(\mathbf{r})=\int G_{i k}\left(\mathbf{r}, \mathbf{r}^{\prime}\right) f_{k}\left(\mathbf{r}^{\prime}\right) d S^{\prime}
$$

where $G_{i k}\left(\mathbf{r}, \mathbf{r}^{\prime}\right)$ is the so called Green's tensor (see, for example, $[16])$. Then, the strain field is determined by:

$\epsilon_{i k}(\mathbf{r})=\frac{1}{2} \int\left(\frac{\partial G_{k m}\left(\mathbf{r}, \mathbf{r}^{\prime}\right)}{\partial x_{i}}+\frac{\partial G_{i m}\left(\mathbf{r}, \mathbf{r}^{\prime}\right)}{\partial x_{k}}\right) f_{m}\left(\mathbf{r}^{\prime}\right) d S^{\prime} .(6)$

This strain is fully defined by the corresponding Green's tensor and Eqs. $(4,5)$. The strain components $\epsilon_{\tau \tau}, \epsilon_{s \tau}, \epsilon_{s s}$ are continuous across the interface while the other components are discontinuous:

$$
\begin{aligned}
& \epsilon_{n n}^{(\beta)}-\epsilon_{n n}^{(\alpha)}=\epsilon_{n n}^{0}+\frac{\nu}{1-\nu}\left(\epsilon_{\tau \tau}^{0}+\epsilon_{s s}^{0}\right), \\
& \epsilon_{n \tau}^{(\beta)}-\epsilon_{n \tau}^{(\alpha)}=\epsilon_{n \tau}^{0}, \quad \epsilon_{n s}^{(\beta)}-\epsilon_{n s}^{(\alpha)}=\epsilon_{n s}^{0} .
\end{aligned}
$$

Taking these jumps of the strain field at the interface into account, one can find the elastic contribution to the local

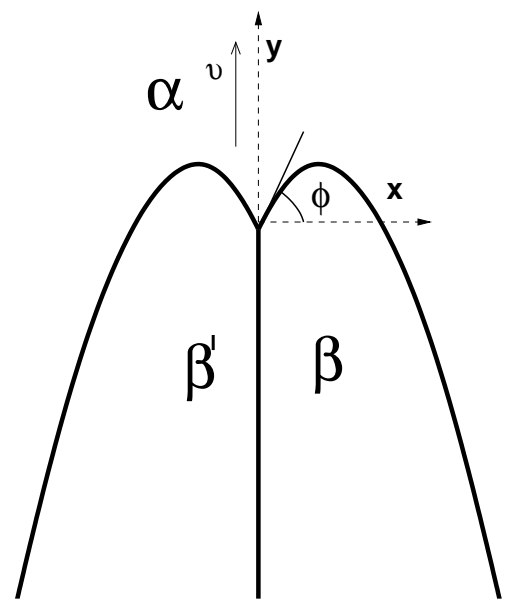

FIG. 1: Steady state growth of a bicrystal $\left(\beta / \beta^{\prime}\right)$. The structure propagates with a constant velocity $v$ along the $y$ axis. The $\beta$-phase corresponds to $\theta=2 \pi / 3$ and the $\beta^{\prime}$-phase corresponds to $\theta=-2 \pi / 3$.

equilibrium condition, Eq. (3). A tedious but straightforward calculation leads to:

$$
\begin{array}{r}
\delta \tilde{F}^{(e l)}=\tilde{F}_{\alpha}^{(e l)}-\tilde{F}_{\beta}^{(e l)}=\sigma_{i k}^{(0)} \epsilon_{i k}^{(\alpha)}- \\
-\frac{E\left[\left(\epsilon_{\tau \tau}^{0}\right)^{2}+\left(\epsilon_{s s}^{0}\right)^{2}+2 \nu \epsilon_{s \epsilon_{\tau \tau}^{0}}^{0}+2(1-\nu)\left(\epsilon_{s \tau}^{0}\right)^{2}\right]}{2\left(1-\nu^{2}\right)}
\end{array}
$$

where $\epsilon_{i k}^{(\alpha)}$ is the strain in the $\alpha$ phase at the interface. Note that the expression above is a complicated integrodifferential functional of the interface shape.

Diffusional growth. For simplicity, we consider transitions in pure materials and assume that the heat diffusion constants are equal in both phases (the so-called symmetrical model). Without loss of generality, we assume that the $\beta$ phase is the low temperature phase. We introduce the dimensionless temperature field $w=c_{p}\left(T-T_{\infty}\right) / L$, where $L$ is the latent heat, $c_{p}$ is heat capacity, and $T_{\infty}$ is the temperature in the $\alpha$ phase far away from the interface. The temperature field $w$ obeys the following heat diffusion equation and boundary conditions

$$
\begin{array}{r}
D \nabla^{2} w=\partial w / \partial t, \\
v_{n}=D \mathbf{n}\left(\left.\nabla w_{\beta}\right|_{\text {int }}-\left.\nabla w_{\alpha}\right|_{\text {int }}\right), \\
\left.w\right|_{\text {int }}=\Delta-d_{0} \kappa+T_{e q} c_{p} \delta \tilde{F}^{e l} / L^{2},
\end{array}
$$

where $d_{0}=\gamma T_{e q} c_{p} / L^{2}$ is the capillarity length, $\kappa$ is the curvature of the interface, which assumed to be positive for convex interfaces, $D$ is the thermal diffusion constant, and $T_{e q}$ is the equilibrium temperature for the flat interface without elastic effects i.e, it is determined by the condition $F_{\alpha}^{0}\left(T_{e q}\right)=F_{\beta}^{0}\left(T_{e q}\right)$. We also introduce the dimensionless undercooling $\Delta=c_{p}\left(T_{e q}-T_{\infty}\right) / L$. The physics underlying Eqs. (8-10) is quite simple. The interface moving with the normal velocity $v_{n}$ releases the 
latent heat. Requirement of heat conservation at the interface gives Eq. (9). The local thermodynamical equilibrium at the interface, Eq. (3), implies Eq. (10), which gives the equilibrium value of the temperature at the interface taking into account the curvature corrections and the elastic effects. The thermal field can be eliminated by using the Green's function techniques (see for example [14]), and consequently together with a proper Green's tensor $G_{i k}\left(\mathbf{r}, \mathbf{r}^{\prime}\right)$ for the elastic field, the set of Eqs. (610) can be incorporated into a single integro-differential equation for the shape of the solid-solid interface.

Dilatation. First, we consider the dilatational case, $\epsilon_{i k}^{0}=\varepsilon \delta_{i k}$, where the bond lengths of the new phase are uniformly longer or shorter in all directions in comparison to the original phase. In this case, the elastic contribution to the local equilibrium condition at the interface, $\delta \tilde{F}^{(e l)}=-\varepsilon^{2} E /(1-\nu)$, is a constant along the interface for any interface shape. This result corresponds to the elastic hysteresis mentioned above and it can be obtained using the analogy of this elastic problem to the problem of thermal expansion for a given temperature field [16]. As a consequence, the equilibrium interface temperature is shifted by a constant value, and the problem is equivalent to the problem of the classical dendritic growth. The anisotropy of the surface energy is a prerequisite for the existence of the dendritic solution in this case, as we have already mentioned above. Note that this exact result is valid only under the assumptions of our model i.e., the isotropic elasticity and equal elastic moduli in both phases. It also serves as a nontrivial check of our numerical code.

Shear transition. Let us consider now a simple type of transition in hexagonal crystals involving the shear strain. For the transitions lowering the symmetry from $C_{6}$ to $C_{2}$ the shear strain in the basic plane appears. This is the case, for example, in hexagonal-orthorhombic transitions in ferroelastics (see [17] and references therein). Let the principal axis $C_{6}$ be orientated in the $z$ direction. We assume that the system obeys a translational invariance in this direction, and thus it is effectively twodimensional. By proper choice of the crystal orientation around the main axis in the initial phase, we obtain the new phase in three possible states, having the following nonvanishing components of the strain tensor $\epsilon_{i k}^{0}$ :

$$
\epsilon_{x x}^{0}=-\epsilon_{y y}^{0}=\varepsilon \cos 2 \theta \quad \epsilon_{x y}^{0}=\varepsilon \sin 2 \theta,
$$

where the angle $\theta$ has three possible values: $\theta=$ $0, \pm 2 \pi / 3$. The situation of $\theta=0$ corresponds to the single crystal growth and $\theta= \pm 2 \pi / 3$ to the bicrystal growth (Fig.1). Because the elasticity of hexagonal crystals is axisymmetric in the harmonic approximation and $\epsilon_{i z}^{0}=\epsilon_{i z}=0$, we can use the isotropic theory of elasticity i.e., expressions for free energy $(1,2)$ remain valid [16]. The moduli of the effective isotropic elasticity, $\lambda$ and $\mu$, can be expressed in terms of the elastic constants of the original hexagonal crystal. The two-dimensional Green's tensor for isotropic materials is [18]:

$$
G_{i k}(\mathbf{r})=\frac{1+\nu}{4 \pi(1-\nu) E}\left(\frac{x_{i} x_{k}}{r^{2}}-(3-4 \nu) \delta_{i k} \ln (r)\right) .
$$

Eliminating the thermal field, we obtain the steady state equation for the shape of the solid-solid interface. In the co-moving frame of reference, this equation reads:

$$
\begin{array}{r}
\Delta-\frac{d_{0} \kappa}{R}+\frac{T_{e q} c_{p} \delta \tilde{F}^{e l}}{L^{2}}= \\
=\frac{p}{\pi} \int d x^{\prime} \exp \left[-p\left(y(x)-y\left(x^{\prime}\right)\right)\right] K_{0}(p \eta),
\end{array}
$$

where $\eta=\left[\left(x-x^{\prime}\right)^{2}+\left(y(x)-y\left(x^{\prime}\right)\right)^{2}\right]^{\frac{1}{2}}$, and $K_{0}$ is the modified Bessel function of third kind in zeroth order, and $p=v R / 2 D$ is the Peclet number. All lengths are reduced by the radius of the curvature $R$ of asymptotic Ivantsov parabola.

In the asymptotic region $(|x| \rightarrow \infty)$, the strain $\epsilon_{i k}$ decays and the local contribution to $\delta \tilde{F}^{e l}$ (second term in Eq. 7) has a constant value, $\delta \tilde{F}^{e l}=-E\left(\epsilon_{y y}^{0}\right)^{2} / 2\left(1-\nu^{2}\right)$. It follows from this relation that the temperature shift (elastic hysteresis) for the growth of a single crystal is four times larger than for a bicrystal. Although both bicrystal configurations $\left(\beta / \beta^{\prime}\right.$ and $\left.\beta^{\prime} / \beta\right)$ are energetically equivalent far from the tip, the symmetry is broken by the choice of the propagation direction. Therefore, in the following we will discuss the most favorable configuration of bicrystals [12], as presented in Fig. 1.

Let us introduce the shifted, due to the elastic hysteresis, undercooling:

$$
\tilde{\Delta}=\Delta-\Delta_{e l}, \quad \Delta_{e l}=T_{e q} c_{p} E \varepsilon^{2} / 8\left(1-\nu^{2}\right) L^{2} .
$$

The dimensionless parameter $\Delta_{e l}$ describes the strength of the elastic effects. The relation between this shifted undercooling $\tilde{\Delta}$ and the Peclet number is given by the two-dimensional Ivantsov formula $[3]: \tilde{\Delta}=$ $\sqrt{p \pi} \exp (p) \operatorname{erfc}(\sqrt{p})$.

The presence of the twin $\left(\beta / \beta^{\prime}\right)$ boundary leads to additional effects. First, calculating the strain field, $\epsilon_{i k}$, which enters in the expression for $\delta \tilde{F}^{(e l)}$, in addition to the integrals along $\alpha / \beta$ and $\alpha / \beta^{\prime}$ interface, the integration should also be performed along the twin boundary $\beta / \beta^{\prime}$. The force density at this boundary is: $f_{x}=0, f_{y}=E \sqrt{3} /(1+\nu)$. Second, the equilibrium angle $\phi$ at the triple junction (Fig. 1) is given by Young's law: $2 \gamma \sin \phi=\gamma_{b}$, where $\gamma$ is the surface energy of the $\beta / \alpha$ interface and $\gamma_{b}$ is the surface energy of the twin boundary.

Eq. (13) is a complicated nonlinear integro-differential equation for the interface shape. We should find a solution of this equation which has a proper angle $\phi$ at the triple junction and which is close to the Ivantsov parabola $\left(y=-x^{2} / 2\right)$ in the tail region. Note that without elastic effects, this problem is equivalent to the classical dendritic growth problem with isotropic surface tension. The 


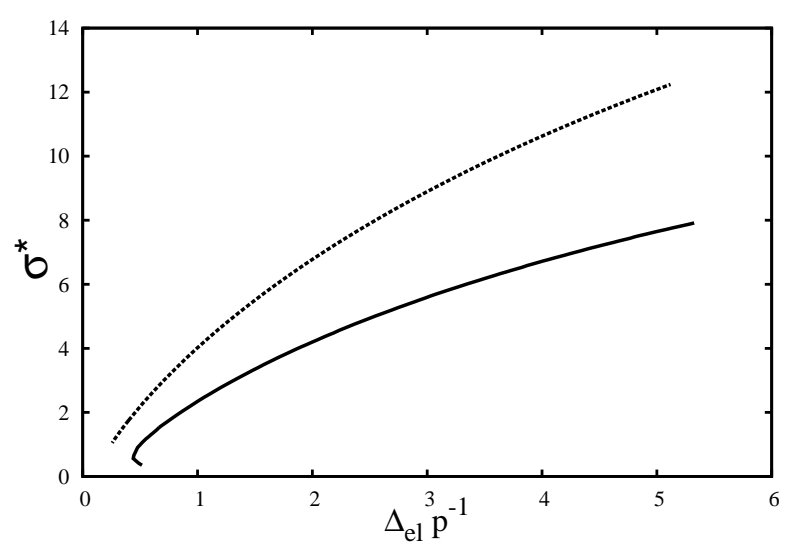

FIG. 2: Stability parameter $\sigma^{*}$ versus $\Delta_{e l} / p$ for two values of $\phi$ : the dashed line corresponds to $\phi=0$, and the solid line corresponds to $\phi=\pi / 6$.

latter does not have a solution with angles $\phi \geq 0[1,2]$. This statement can be expressed in the following form. For any given positive values of the Peclet number $p$ and the so-called stability parameter $\sigma=d_{0} / p R$, the symmetric solution which is close to the Ivantsov parabola in the tail region has an angle at the tip $\phi=f(\sigma, p)<0$. The limit $\sigma=0$ and $\phi=0$ is a singular limit for that problem. For example, Meiron [19] calculated the angle $\phi$ as a function of $\sigma$ for several values of the Peclet number with isotropic surface tension numerically and found that the angle $\phi<0$ for any positive $\sigma$.

Now, we discuss the numerical results obtained by the solution of Eq. (13) in the spirit of Ref. [19]. In the important regime of small Peclet numbers, the eigenvalue $\sigma=\sigma^{*}\left(\phi^{*}, \Delta_{e l}, p\right)$ depends only on the ratio $\Delta_{e l} / p$ for a fixed angle $\phi$. While the strength of the elastic effects is assumed to be small, $\Delta_{e l} \ll 1$, the control parameter $\Delta_{e l} / p$ can be varied in a wide region in the limit of small $p$. The eigenvalue $\sigma^{*}$ as a function of $\Delta_{e l} / p$ for two values of the angle, $\phi=0$ and $\phi=\pi / 6$, is shown in Fig.2. The situation with $\phi \approx 0$ is realized if $\gamma_{b} \ll \gamma$, while $\phi \approx \pi / 6$ corresponds to $\gamma_{b} \approx \gamma$. The Poisson ratio was fixed to $\nu=1 / 3$.

The most remarkable feature of these results is that we do find dendritic solutions for the isotropic surface tension in the presence of the elastic effects. In this sense, the elastic effects serve as a new selection mechanism. We note that $\sigma^{*}$ becomes large for large values of $\Delta_{e l} / p$, while in the classical dendritic growth $\sigma^{*}$ is always small, being controlled by tiny anisotropy effects. Thus, the growth velocity, $v=2 D \sigma^{*} p^{2} / d_{0}$, can be much larger due to elastic effects, compared to the classical dendritic growth.

In the case $\phi=\pi / 6$, the solution exists only beyond some critical value of the control parameter, $\Delta_{e l} / p$. The lower branch of this solution has a negative curvature near the triple junction and it is presumably unstable. The general structure of the theory suggests that in the case $\phi=0$, the curve should start from the origin $(\sigma=$ $\left.0, \Delta_{e l} / p=0\right)$ which is a singular point of the problem. However, numerics becomes very difficult in the vicinity of this point.

We have also performed several runs for the single crystal growth $(\theta=0$ in Eq. (11)). As in the classical dendritic growth, we have not found solutions with a smooth tip, $\phi=0$. Moreover, for negative values of $\phi$, where solutions exist and correspond to the growth along a grain boundary in the mother $\alpha$ phase [20], the selected stability parameter $\sigma^{*}$ is a decreasing function of $\Delta_{e l} / p$. This is in strong contrast with the results for the bicrystal growth.

We hope that our results will stimulate new experimental and theoretical investigations in this interesting field. On the theoretical side, a challenging problem would be the investigation of the doublon patterns in the case of bicrystal growth. With this scenario, two crystals of the $\beta$ phase would be separated by a thin film of the mother $\alpha$ phase and not by the twin boundary. This pattern is a competitive structure at least in the range of the small control parameter $\Delta_{e l} / p$, where the dendritic pattern with the twin boundary does not exist.

This work has been supported in part by the Deutsche Forschungsgemeinschaft under Grant SSP 1296 and by the German-Israeli Foundation.

[1] D. Kessler, J. Koplik, H. Levine, Adv. Phys. 37 , 255 (1988).

[2] E. A. Brener, and V. I. Mel'nikov, Adv. Phys. 4053 (1991)

[3] G. P. Ivantsov, Dokl. Akad. Nauk USSR 58, 567 (1947).

[4] E. Brener, Phys. Rev. Lett. 71, 3653 (1993).

[5] M. Ben Amar and E. Brener, Phys. Rev. Lett. 71, 589 (1993).

[6] M. Ben Amar and E. Brener, Phys. Rev. Lett. 75, 561 (1995).

[7] T. Ihle and H. Müller-Krumbhaar, Phys. Rev. Lett. 70, 3083 (1993).

[8] A. L. Roitburd, Sov. Phys. Usp. 17, 326 (1974).

[9] A. G. Khachaturyan. Theory of Structural Transformation in Solids (Wiley, New York, 1983).

[10] E.A. Brener and V. I. Marchenko, JETP Lett. 56, 368 (1992)

[11] E.A. Brener, S. V. Iordanskii, and V. I. Marchenko, Phys. Rev. Lett. 82, 1506 (1999).

[12] E.A. Brener, V. I. Marchenko, and R. Spatschek, Phys. Rev. E 75, 041604 (2007).

[13] J. Slutsker, K Thornton, A. L. Roitburd, J. A. Warren and G.B. McFadden, Phys. Rev. B 74014103 (2006).

[14] J. S. Langer, Rev. Mod. Phys. 52, 1 (1980)

[15] I.A. Privorotskii, Sov. Phys. JETP 33, 8251971.

[16] L. D. Landau and E. M. Lifshitz, Theory of Elasticity Pergamon Press, Oxford, (1987). 
[17] S. H. Curnoe and A. E. Jacobs, Phys. Rev. B 63. 094110 (2001).

[18] C. A. Brebbia, J. Dominguez, Boundary Elements, An Introductory Course. McGraw-Hill, (1989)
[19] D. I. Meiron, Phys. Rev. A 33, 2704 (1986)

[20] E. A. Brener, C. Hüter, D. Pilipenko, and D. E. Temkin, Phys. Rev. Lett. 99, 105701 (2007). 